\title{
High level in vivo mucin-type glycosylation in Escherichia coli
}

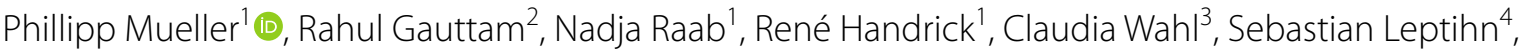 \\ Michael Zorn ${ }^{5}$, Michaela Kussmaul ${ }^{5}$, Marianne Scheffold ${ }^{5}$, Bernhard Eikmanns' ${ }^{2}$, Lothar Elling ${ }^{3}$ \\ and Sabine Gaisser ${ }^{1 *}$
}

\begin{abstract}
Background: Increasing efforts have been made to assess the potential of Escherichia coli strains for the production of complex recombinant proteins. Since a considerable part of therapeutic proteins are glycoproteins, the lack of the post-translational attachment of sugar moieties in standard E. coli expression strains represents a major caveat, thus limiting the use of E. coli based cell factories. The establishment of an E. coli expression system capable of protein glycosylation could potentially facilitate the production of therapeutics with a putative concomitant reduction of production costs.
\end{abstract}

Results: The previously established E. coli strain expressing the soluble form of the functional human-derived glycosyltransferase polypeptide N-acetylgalactosaminyltransferase 2 (GalNAc-T2) was further modified by co-expressing the UDP-GlcNAc 4-epimerase WbgU derived from Plesiomonas shigelloides. This enables the conversion of uridine $5^{\prime}$-diphospho-N-acetylglucosamine (UDP-GlcNAc) to the sugar donor uridine $5^{\prime}$-diphospho- $N$-acetylgalactosamine (UDP-GalNAc) in the bacterial cytoplasm. Initially, the codon-optimised gene wbg $U$ was inserted into a pET-derived vector and a Tobacco Etch Virus (TEV) protease cleavable polyhistidine-tag was translationally fused to the C-terminus of the amino acid sequence. The 4-epimerase was subsequently expressed and purified. Following the removal of the polyhistidine-tag, WbgU was analysed by circular dichroism spectroscopy to determine folding state and thermal transitions of the protein. The in vitro activity of WbgU was validated by employing a modified glycosyltransferase assay. The conversion of UDP-GICNAc to UDP-GalNAc was shown by capillary electrophoresis analysis. Using a previously established chaperone pre-/co- expression platform, the in vivo activity of both glycosyltransferase GalNAc-T2 and 4-epimerase WbgU was assessed in E. coli, in combination with a mucin 10-derived target protein. Monitoring glycosylation by liquid chromatography electrospray ionization mass spectrometry (LC-ESI-MS), the results clearly indicated the in vivo glycosylation of the mucin-derived acceptor peptide.

Conclusion: In the present work, the previously established E. coli- based expression system was further optimized and the potential for in vivo O-glycosylation was shown by demonstrating the transfer of sugar moieties to a mucinderived acceptor protein. The results offer the possibility to assess the practical use of the described expression platform for in vivo glycosylations of important biopharmaceutical compounds in E. coli.

Keywords: Protein expression, Escherichia coli, Glycosyltransferase GalNAcT2, Chaperone co-expression, In vivo glycosylation, UDP-GlcNAc 4-epimerase WbgU, Mucin-type O-glycosylation

\footnotetext{
*Correspondence: gaisser@hochschule-bc.de

${ }^{1}$ Institute of Applied Biotechnology, Biberach University of Applied

Sciences, Biberach, Germany

Full list of author information is available at the end of the article
} 


\section{Background}

Since the first approval of the recombinantly expressed human therapeutic protein Humulin ${ }^{\circledR}$ in 1982, Escherichia coli has served as reliable and cost-efficient production host in the pharmaceutical industry [1]. In recent years the requirements of modern biopharmaceuticals have shifted towards higher complexity, while consistently maintaining high product quality [2]. Over the years, the lack or insufficient capability of $E$. coli strains to perform post-translational modifications, including disulfide bond formation and glycosylations [2, 3], has contributed to the rise of mammalian rather than nonmammalian expression systems for the commercial production of biopharmaceutical products [4]. However, as a very popular expression host, $E$. coli remains an important factor in biopharmaceutical manufacturing [4]. To enable recombinant expression of complex proteins in $E$. coli, research has focused on improving protein folding, disulfide bond formation, as well as $N$ - and $O$-linked glycosylation [5-11]. $N$-linked glycosylation in $E$. coli has been achieved by transferring the $p g l$ gene cluster derived from Campylobacter jejuni into $E$. coli with the subsequent expression of glycosyltransferases and enzymes required for sugar biosynthesis in the strain background [10-12]. The glycan is assembled on a membraneanchored precursor molecule and flipped into the periplasm, where the oligosaccharyltransferase PglB attaches the glycan onto the target protein [12]. However, the glycosylation site consensus sequence of PglB is more stringent than the eukaryotic polypeptide acceptor sequence. As a result, engineering of the polypeptide sequence of the target protein or altering the substrate specificity of PglB is required to promote correct $N$-glycosylation of recombinant proteins expressed in the periplasm of $E$. coli [13]. In contrast, $O$-glycosylation of eukaryotic target sites using a truncated form of the human glycosyltransferase GalNAc-T2 has been shown without altering the target peptide sequence $[6,9,14,15]$.

$O$-glycosylation involves the transfer of sugar moieties to a serine or threonine residue, commonly found on mucins. Mucins are cell surface or secreted highmolecular weight glycoproteins on the mucosal epithelium in animals and humans, which provide a protective shield against toxins and pathogens $[3,16]$. $O$-glycosylation of mucins is initiated by the transfer of GalNAc-a reaction catalyzed by UDP- $N$-Acetylgalactosaminyltransferases (GalNAc-Ts)-forming the GalNAc $\alpha 1-O$-serine or threonine linkage [16]. Different mucin-derived target peptides have been successfully used as substrates to assess the activity of the human glycosyltransferase GalNAc-T2 lacking the $\mathrm{N}$-terminal transmembrane domain $[6,9,14,15]$. As an example, glycosylation of the synthetic peptide EA2
(PTTDSTTPAPTTK)—derived from Rattus norvegicus submandibular apomucin 10 (MUC10)-has been demonstrated in various in vitro assays in the presence of soluble GalNAc-T2 [6, 14, 15].

The in vivo $O$-glycosylation of a glutathione S-transferase-mucin peptide fusion in an E. coli SHuffle ${ }^{\circledR} \mathrm{T} 7$ express strain co-expressing an $\mathrm{N}$-terminal truncated version of GalNAc-T2, in combination with the UDPGlcNAc 4-epimerase WbpP from Pseudomonas aeruginosa has been indicated based on Western blot analysis using horseradish peroxidase-conjugated Vicia villosa lectin [9]. However, the detailed analysis of the glycosylated target mucin peptide with eight potential glycosylation sites has not been shown [9]. Additionally, potential $O$-glycosylation sites comprising eight amino acids of relevant biopharmaceuticals have been fused to anti-TNF- $\alpha$ Fab and analysed for the presence of GalNAc $\alpha 1-O$-serine or threonine [9].

As a first step to establish an $E$. coli-based expression platform enabling the glycosylation of full-length biopharmaceuticals in vivo, an active human GalNAcT2 derivative comprising amino acids 52-571 with a translationally fused $\mathrm{N}$-terminal HisDap (diamino peptidase) tag has been isolated from a SHuffle ${ }^{\circledR}$ T7 E. coli strain pre-/co-expressing chaperones [6]. The activity of the purified glycosyltransferase has been demonstrated using the mucin peptide derivative EA2 and filgrastim (Granulocyte-colony stimulating factor, G-CSF) as acceptor substrates in vitro [6]. As a further step to develop an E. coli expression strain for the production of glycosylated biopharmaceuticals, the presented approach describes the addition of the active Plesiomonas shigelloides-derived UDP-GlcNAc 4-epimerase (WbgU), to ensure the presence of the sugar substrate UDP-GalNAc in the E. coli strain background. WbgU was chosen based on the well-characterized properties of the enzyme [17]. An artificial protein derived from MUC10 (GenBank: AAA20966.1, UniProtID: Q62605) was used as the target protein. In the present work, we successfully show in vivo glycosylation by co-expressing the 4-epimerase $\mathrm{WbgU}$, the truncated form of the glycosyltransferase GalNAc-T2, and the MUC10 target protein in the established E. coli system pre-/coexpressing the chaperones sulfhydryl oxidase Erv1p (essential for respiration and vegetative growth protein 1 ) and the human protein disulfide isomerase PDI, as previously described $[6,8]$. The presence of functional GalNAc-T2, WbgU and soluble expressed MUC10 derivative was demonstrated by immunoblot analysis. WbgU was detected with polyclonal antibodies in serum samples taken from rabbits immunized with purified protein. The detailed analysis of the in vivo glycosylated target protein is shown. 


\section{Results}

\section{Expression, purification, and analysis of $\mathrm{WbgU}$}

Experiments to express the 4-epimerase were performed using E. coli host strain SHuffle ${ }^{\circledR} \mathrm{T} 7$ Express harbouring plasmids pMJS9 and pET23a $(+) \_w b g U T E V 6 H$ as previously described $[6,8]$. The specific cleavage sequence for the Tobacco Etch Virus nuclear-inclusiona endopeptidase (TEV protease) inserted between the WbgU amino acid sequence and translationally fused C-terminal polyhistidine tag $(6 \mathrm{H}$, His-tag) was used to obtain tag-free enzyme via affinity chromatography and subsequent protease treatment (Fig. 1a, b). Soluble expression of WbgUTEV6H (41 kDa) was found to be best in Terrific Broth (TB) medium without Isopropyl$\beta$-D-thiogalactopyranosid (IPTG) induction and with the pre-/co-expression of the redox folding helper proteins Erv1p and PDI encoded by pMJS9 under the control of an arabinose promotor (data not shown). Soluble WbgUTEV6H (Fig. 1b, lane S) was purified by employing Ni-NTA affinity chromatography. The protein was clearly visible in eluted fractions (Fig. 1a, b, lanes E2E6), which were pooled and treated with polyhistidinetagged TEV protease. The cleaved His-tag and TEV protease were removed in a subsequent affinity chromatography step and $\mathrm{WbgU}$ identified by $\mathrm{N}$-terminal Edman degradation. The tag-free protein migrated at $40 \mathrm{kDa}$ under reducing denaturing conditions in the SDS-gel and was no longer detected using Anti-His antibodies (Fig. 1a, b, lanes F1 and F2). The protein was further used for the immunization of rabbits to generate polyclonal antibodies for the detection of $\mathrm{WbgU}$ in immunoblot assays. The obtained sera samples were assessed and immunoblot conditions were optimized to minimize unspecific signals (data not shown).

\section{Circular dichroism (CD) spectrum analysis}

The secondary structure and thermal transition points of WbgU were assessed by CD spectroscopy (Fig. 2). Characteristic minima at 208 and $222 \mathrm{~nm}$, typical for $\alpha$-helical structures, were identified in CD scans indicating the folding of $\mathrm{WbgU}$ (Fig. 2a). The slight difference in the scans measured at $20{ }^{\circ} \mathrm{C}$ and $37{ }^{\circ} \mathrm{C}$ potentially indicates some reduction of secondary structures at the higher temperature. However, it has already been shown by Kowal and Wang, that WbgU exhibits the highest activity at $37{ }^{\circ} \mathrm{C}$ [17]. The thermal unfolding profiles of $\mathrm{WbgU}$ were monitored by recording temperature-dependent molar ellipticity changes at $220 \mathrm{~nm}$ from $20{ }^{\circ} \mathrm{C}$ to $70{ }^{\circ} \mathrm{C}$. The thermal transition points at $42.55 \pm 0.04{ }^{\circ} \mathrm{C}$, increasing to $43.07 \pm 0.04{ }^{\circ} \mathrm{C}$, and $51.29 \pm 0.13{ }^{\circ} \mathrm{C}$ in the presence of UDP-GlcNAc and UDP-GalNAc, respectively, were determined based on the sigmoid graphs displayed in Fig. 2b. We concluded that the increase detected in samples containing UDP-GalNAc may indicate a stabilizing effect in the presence of the sugar substrate. Similarly, an enhancement of the conformational stability has been postulated for the human UDP-galactose $4^{\prime}$-epimerase GALE when binding the substrate UDP-glucose and the authors hypothesized that natural ligands modulate GALE stability in vivo [18].

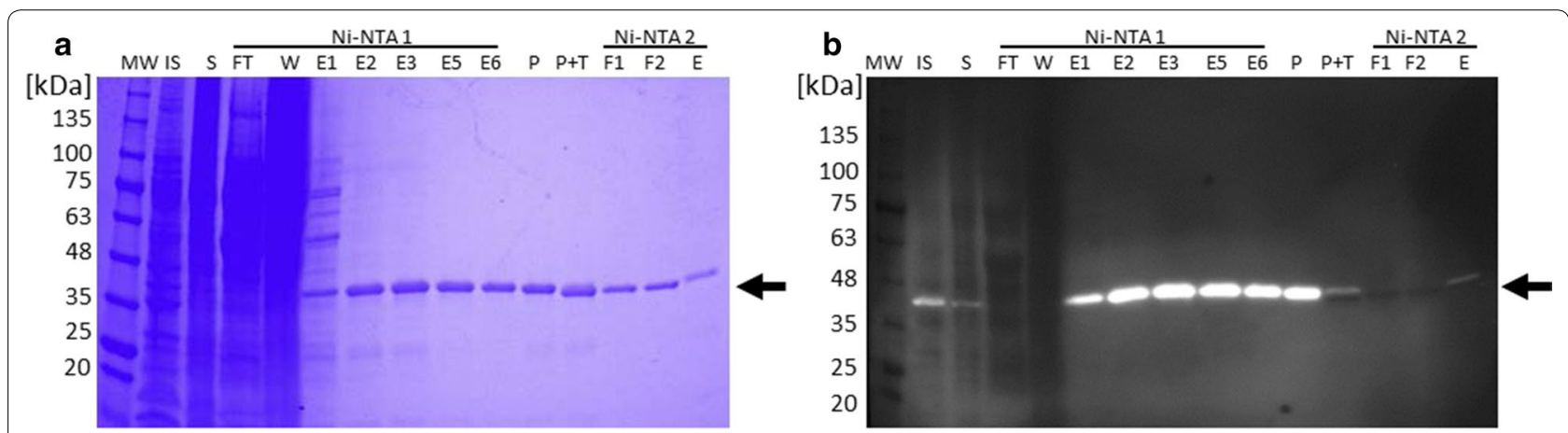

Fig. 1 SDS-PAGE (a) and immunoblot analysis (b) of WbgUTEV6H expression in E. coli and purification of WbgU. E. coli SHuffle ${ }^{\circledR}$ T7 Express cells carrying plasmids pET23a(+)_wbgUTEV6H and pMJS9 were grown in TB medium at $30^{\circ} \mathrm{C}$ until $\mathrm{OD}_{600}$ of 1 was reached. L-arabinose was added to a final concentration of $0.5 \%$ and the culture was incubated for another $6.5 \mathrm{~h}$. The cells were harvested by centrifugation, lysed, and insoluble particulate fractions (IS) and soluble fractions (S) were isolated. WbgUTEV6H was purified from the soluble fraction using Ni-NTA chromatography (Ni-NTA 1). Fractions of the flow-through (FT), washing (W), and elution (E1-E6) were collected. Fractions E2-E6 were pooled (P) and incubated with TEV protease $(P+T)$. The cleaved His-tag, TEV protease, and undigested WbgUTEV6H were removed using a second affinity chromatography step (Ni-NTA 2, E). Aliquots $(10 \mu l)$ of the samples and collected fractions were separated by SDS-PAGE and visualized by Coomassie staining (a) and immunoblotting (b). WbgUTEV6H with an estimated mass of $41 \mathrm{kDa}$ was detected using mouse Anti-His.H8 antibodies. Tag-free WbgU (40 kDa) was clearly visible in the flow-through (a, F1 and F2). The respective bands were no longer detected in the immunoblot assay with Anti-His.H8 antibodies (b, F1 and F2) indicating the successful removal of the polyhistidine tag. Molecular mass markers (MW) are in kDa 

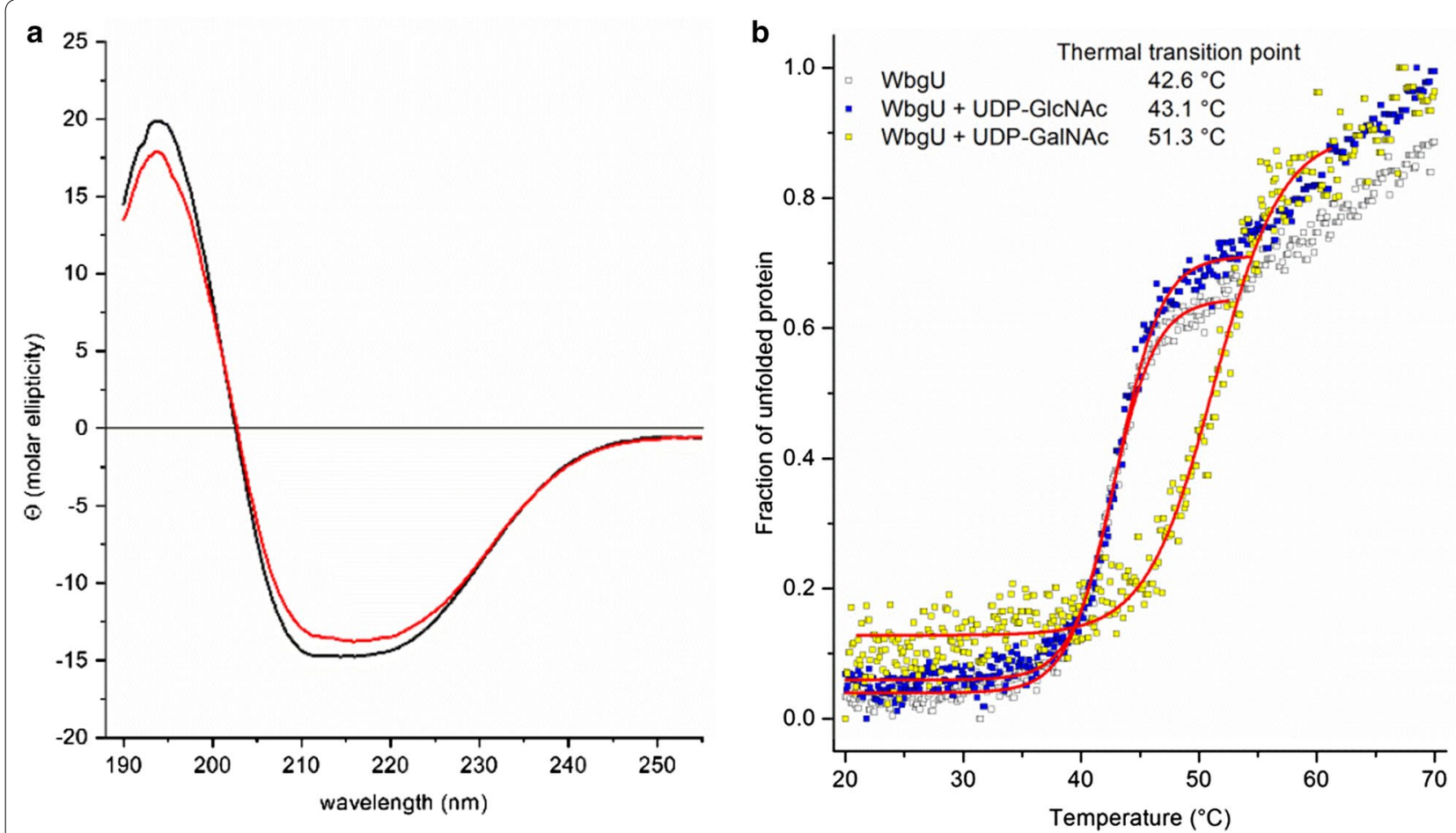

Fig. $2 \mathrm{CD}$ spectroscopy and thermal stability measurements of WbgU. a Far UV (195-260 nm) spectra of WbgU at $20^{\circ} \mathrm{C}$ (black line) and $37^{\circ} \mathrm{C}($ red line) indicating the folding status of the 4-epimerase in $20 \mathrm{mM}$ sodium phosphate buffer at $\mathrm{pH} 7.75$. CD data were reported as molar ellipticity $(\Theta)$. b Thermal unfolding profiles of WbgU (white squares), WbgU in the presence of either UDP-GICNAc (blue squares) or UDP-GalNAc (yellow squares) were monitored at $220 \mathrm{~nm}$

\section{Analysis of WbgU enzymatic activity}

The equilibrium reaction between UDP-GalNAc and UDP-GlcNAc in the presence of WbgU [17] was assessed by monitoring the conversion of the substrates at concentrations of $0.15 \mathrm{mM}$ or $1.5 \mathrm{mM}$. After heat inactivation of WbgU, UDP-GalNAc and UDP-GlcNAc were separated by capillary electrophoresis and detected at $254 \mathrm{~nm}$. When $0.15 \mathrm{mM}$ UDP-sugar substrates were used, the results showed a ratio of 30:70 of UDP-GalNAc:UDP-GlcNAc (Fig. 3a). In samples containing $1.5 \mathrm{mM}$ UDP-GlcNAc, a substrate conversion of about $30 \%$ was detected, whereas $57 \%$ of $1.5 \mathrm{mM}$ UDP-GalNAc was converted during the reaction time, indicating a faster conversion of UDP-GalNAc (Fig. 3a). These results indicate the successful expression of functional WbgU of $P$. shigelloides in E. coli $\mathrm{SHuffl}^{\circledR} \mathrm{T} 7$ Express. These results also correlate with data previously published for the His-tagged 4-epimerase WbgU recombinantly expressed in E. coli BL21(DE3) [17]. In the cited paper $K_{\mathrm{m}}$ values of $0.13 \mathrm{mM}$ are reported for both substrates. At substrate saturation $(1.5 \mathrm{mM})$, reaction velocities are different with the respective starting substrate [17]. The authors have described a slower conversion rate of UDP-GlcNAc than that of
UDP-GalNAc based on time course experiments using multiple enzyme dilutions [17].

The in vitro activity of the epimerase combined with the commercially available glycosyltransferase rhGalNAc-T2 and purified recombinant HisDapGalNAc-T2 from $E$. coli, was assessed using a modified glycosyltransferase assay [6]. The assay is based on the detection of inorganic phosphate, which is released during the sugar transfer reaction and subsequent hydrolysis of UDP catalyzed by a phosphatase. The released phosphate is optically quantified using malachite green reagents [19]. In order to combine the epimerization reaction and the glycosyltransferase assay, a pre-incubation step of the glycosyltransferase reaction mix containing the sugar substrate and the acceptor peptide with $\mathrm{WbgU}$ was added. The pre-incubation step was optimised assessing different parameters, such as incubation time and enzyme concentration, to minimize a potential limitation of the glycosylation reaction by WbgU. Both glycosyltransferases were assessed in combination with either $0.5 \mathrm{mM}$ UDP-GalNAc or UDP-GlcNAc as substrates, and the determined specific activities are shown in Fig. 3b. Adding WbgU and UDP-GlcNAc instead of UDP-GalNAc, the in vitro activity of $\mathrm{WbgU}$ was demonstrated based 

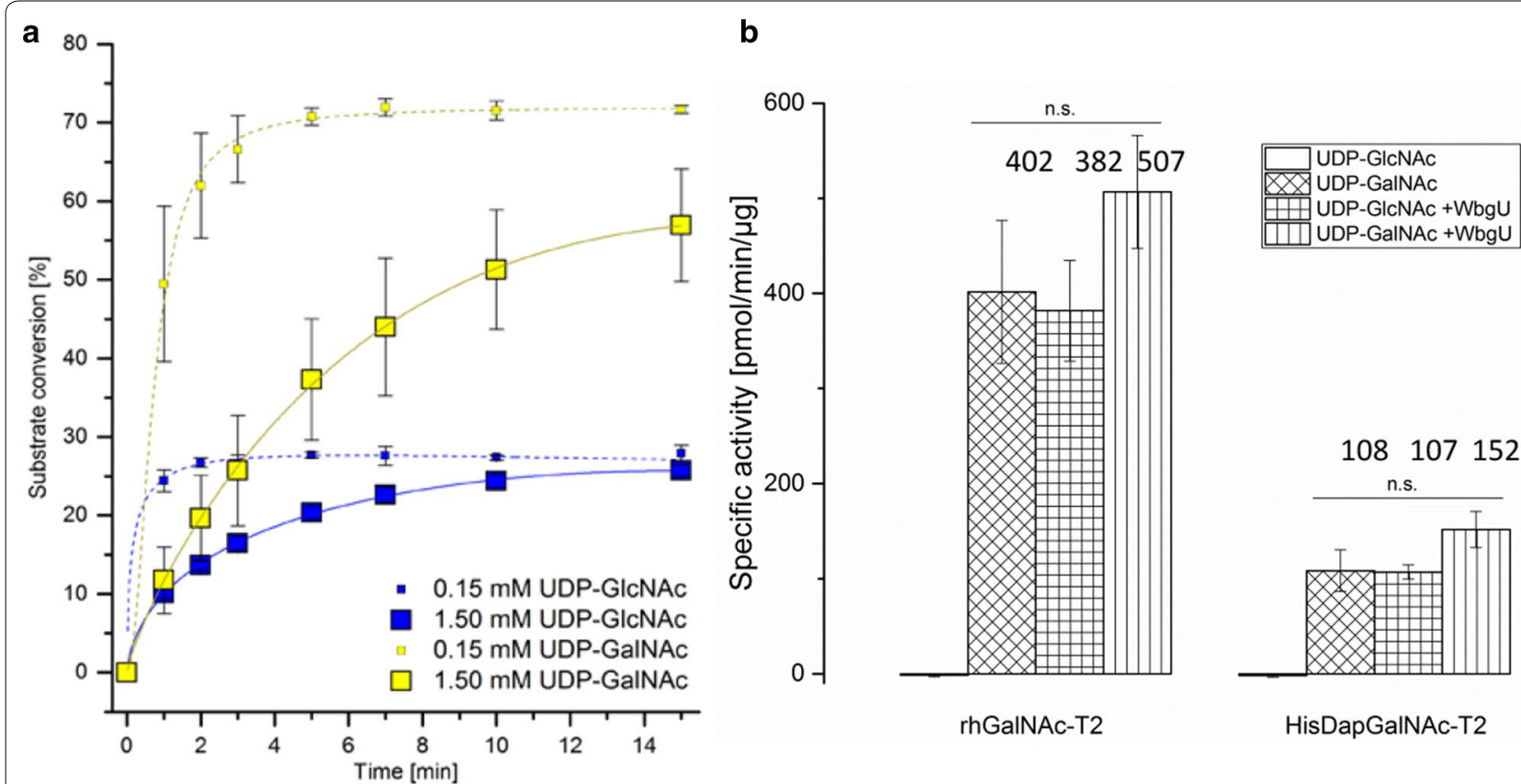

Fig. 3 In vitro activity of WbgU analysed by capillary electrophoresis (CE) (a) and in combination with GalNAc-T2 using a modified glycosyltransferase assay (b). Substrate conversion of the sugar substrates UDP-GIcNAc (blue squares) and UDP-GalNAc (yellow squares) using $0.15 \mathrm{mM}$ (small squares) and $1.50 \mathrm{mM}$ (big squares) in the presence of WbgU. UDP-GIcNAc and UDP-GalNAc were separated by CE and detected at $254 \mathrm{~nm}$. The mean substrate conversion was calculated based on the relative peak area of three independent experiments and results are shown including the standard deviation (a). The mean specific activities of recombinant human GalNAc-T2 (rhGalNAc-T2) commercially produced in NSO cells and His-tagged GalNAc-T2 (HisDapGalNAc-T2) expressed in E. coli were determined in four independent experiments as duplicates using $0.5 \mathrm{mM}$ UDP-GalNAc and UDP-GICNAc, as activated sugar donors, $0.25 \mathrm{mM} \mathrm{EA2}$ peptide acceptor substrate and $1 \mu \mathrm{g}$ WbgU. Samples containing WbgU were incubated for $20 \mathrm{~min}$ at $37^{\circ} \mathrm{C}$ prior to glycosyltransferase addition and subsequently incubated for $20 \mathrm{~min}$ at $37^{\circ} \mathrm{C}$. Results are displayed including the standard error; inserted numbers indicate the specific activities in pmol* $\mathrm{min}^{-1 *} \mathrm{ug}^{-1}$ (b). n.s. not significant

on the release of phosphate in the glycosylation reaction, which indicates the conversion of UDP-GlcNAc to UDPGalNAc. Glycosyltransferase combined with $\mathrm{WbgU}$ and UDP-GalNAc was included as control. In the presence of UDP-GalNAc sugar substrate, the addition of WbgU increased the specific activity of rhGalNAc-T2 and HisDapGalNAc-T2 by about 26 and $40 \%$, respectively. No activity was detected for both glycosyltransferases in the presence of UDP-GlcNAc without WbgU. Specific activities with UDP-GalNAc as a sugar substrate were roughly comparable to the values detected for the combination of WbgU and UDP-GlcNAc. In general, significantly higher activities were detected for rhGalNAc-T2 as compared to HisDapGalNAc-T2 (Fig. 3b), thus confirming previously published results [6].

\section{Co-expression of GaINAc-T2, WbgU, and T7Muc10}

GalNAc-T2, WbgU, and protein target T7Muc10 were expressed simultaneously in a previously established $E$. coli expression system pre-/co- expressing the chaperones Erv1p and PDI, in order to probe the in vivo O-glycosylation activity of GalNAc-T2. The design of the target protein T7Muc10 was based on the amino acid sequence
127 to 230 of rat apomucin, resulting in a protein that comprises six repeats of the EA2 sequence (PTTDSTTPAPTTK) and two repeats of the EAN sequence (PTTDSTTPAPTNK) followed by an asparagine-glycine linker, a thrombin cleavage site, and a $10 \times$ polyhistidine-tag (Fig. 4). As a result of the employed cloning strategy, the T7 leader sequence derived from the pET23d DNA sequence was translationally fused to the $\mathrm{N}$-terminus leading to the expression of T7Muc10 (Fig. 4). Expression experiments were performed including $E$. coli SHuffle ${ }^{\circledR}$ T7 Express pMJS9 strains harbouring either the pET23d vector without insert, a vector construct encoding GalNAc-T2 and T7Muc10 or an expression plasmid carrying the genetic information for the glycosyltransferase, the 4-epimerase and the target protein. Insoluble and soluble fractions derived from lysed cells were analysed by SDS-PAGE (Fig. 5a) and immunoblot analysis (Fig. 5b-d). The expression of GalNAc-T2 $(60 \mathrm{kDa})$ and WbgU (40 kDa) was detected in both insoluble and soluble fractions (GalNAc-T2; Fig. 5b green arrows; WbgU, Fig. 5c blue arrows). Based on densitometric analysis of the Western Blot the amount of soluble WbgU was estimated at around $65 \%$ (Fig. 5c lane 7) in comparison to 


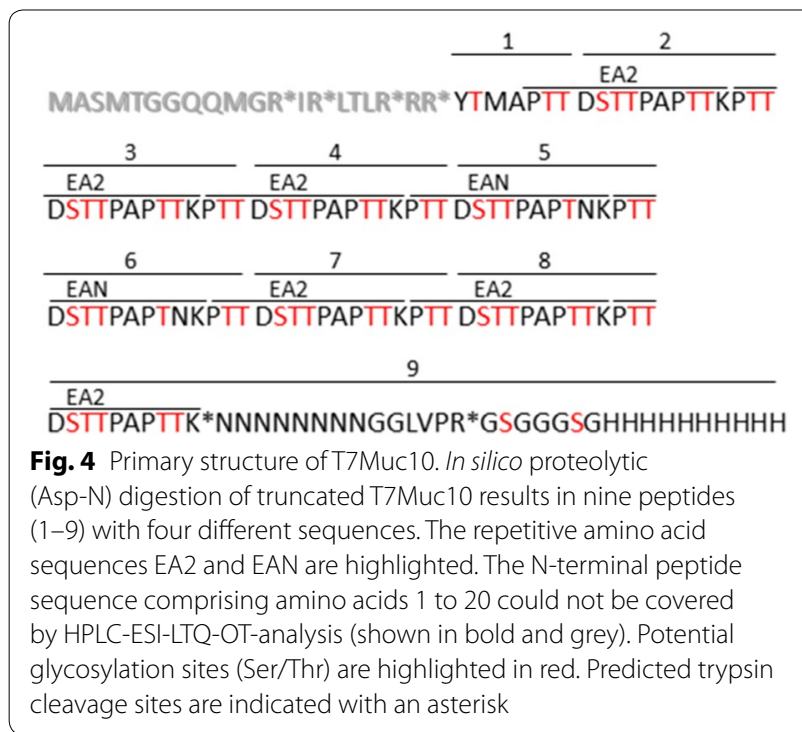

the corresponding fraction of $35 \%$ in the insoluble fraction (Fig. 5c lane 3). However, expressed GalNAc-T2 in the soluble fractions was less prominent (about 33\% as compared to $67 \%$ in the insoluble fractions for both E. coli strains) (Fig. 5b). The detected molecular weight of $32 \mathrm{kDa}$ for T7Muc10 was higher than the expected $16.5 \mathrm{kDa}$, indicating a potential dimerization of the protein (Fig. 5d red arrows). Interestingly, the apparent molecular mass of T7Muc10 increased significantly in the presence of $\mathrm{WbgU}$ (Fig. 5d orange arrows) suggesting a modification by glycosylation.

\section{Analysis of in vivo glycosylation of T7Muc10}

First attempts to capture T7Muc10 using Ni-NTA affinity chromatography failed due to unspecific binding of host cell proteins and weak interaction between protein and column (data not shown). However, the estimated high isoelectric point of the protein $(\mathrm{pI}=10.1)$ allowed for an indirect anion exchange chromatography step (AIEX), thus avoiding the addition of salt and an additional dialysis step to separate the majority of host cell proteins from T7Muc10. Fractions containing prepurified mucin derivatives were subjected to affinity chromatography and the concentrated eluates contained T7Muc10 with sufficient purity for further analysis (Fig. 6b, lanes E1 and E2). The diffuse, slightly smeared protein signal between 33 and $60 \mathrm{kDa}$ detected by Western Blot analysis (Fig. 6, lanes E1 and E2) indicates the presence of putative glycosylated T7Muc10.

To verify the presence of T7Muc10 and determine potential glycosylations in the samples, Coomassiestained protein bands containing either unglycosylated $(33 \mathrm{kDa})$ or the putative glycosylated T7Muc10 (33 kDa to $60 \mathrm{kDa}$ ) were cut from the SDS-polyacrylamide gel. These were submitted for analysis by liquid chromatography electrospray ionization tandem mass spectrometry (LC-ESI-MS/MS). The solubilized protein was treated with trypsin prior to analysis. The detected peptides were assigned and the results indicate the presence of GalNAc-modified threonines in T7Muc10 (Table 1). Evaluation of the tandem MS data provided information about the glycosylation sites targeted by the glycosyltransferase and modified threonines (displayed as lower-case letters in bold type). Positions 3, 7, 11 and 12 of the repetitive EA2 subsequence PTtDSTtPAPttK in T7Muc10 were identified as possible glycosylation sites (Table 1).

To examine the protein expression and overall glycosylation of T7Muc10, samples containing unglycosylated and potentially glycosylated T7Muc10 were subjected to intact mass determination by ultra-performance liquid chromatography coupled with electrospray ionizationquadrupole time-of-flight mass spectrometry (UPLCESI-QTOF-MS). The intact mass analysis revealed an average molecular mass of T7Muc10 of 14,230 Da, instead of the expected 16,531 Da calculated from its theoretical amino acid sequence (Fig. 7a). This result corresponds to a truncated version of T7Muc10 (AS 21 to 159), lacking the first $20 \mathrm{~N}$-terminal amino acids measured with a mass deviation of $68 \mathrm{ppm}$. The presence of truncated recombinantly expressed T7Muc10 has been confirmed by determining amino acids 1 to 5 using $\mathrm{N}$-terminal Edman sequencing. Whether the absence of the $\mathrm{N}$-terminal region was due to cleavage by proteases in the E. coli cytoplasm during expression or a result of proteolytic degradation during purification procedures is still unclear. LC-MS analysis of potentially glycosylated T7Muc revealed different glycosylation stages, ranging from zero up to 24 attached sugar moieties (Fig. 7b). Each attached moiety adds 203.2 Da in mass, which is consistent with the presence of GalNAc. Up to 24 of the 57 potential $\mathrm{O}$-glycosylation sites of the $\mathrm{N}$-terminally truncated T7Muc10 (AS 21-159) were glycosylated, increasing the average molecular mass by roughly $4877 \mathrm{Da}$.

To assess the glycosylation level of EA2- and EAN repetitions, unglycosylated and potentially glycosylated T7Muc10 samples were treated with endoproteinase Asp-N (Fig. 4). These samples were analysed by HPLCESI linear trap quadrupole-orbitrap (LTQ-OT)-MS and MS/MS. Different glycosylation levels of EA2- and EAN-peptides ranging from zero to four attached GalNAc moieties were detected in the MS data (Table 2). Some peptides with the same molecular mass and consequently equal glycosylation level were detected at different retention times indicating different localizations of the attached GalNAc moieties (Table 2). 


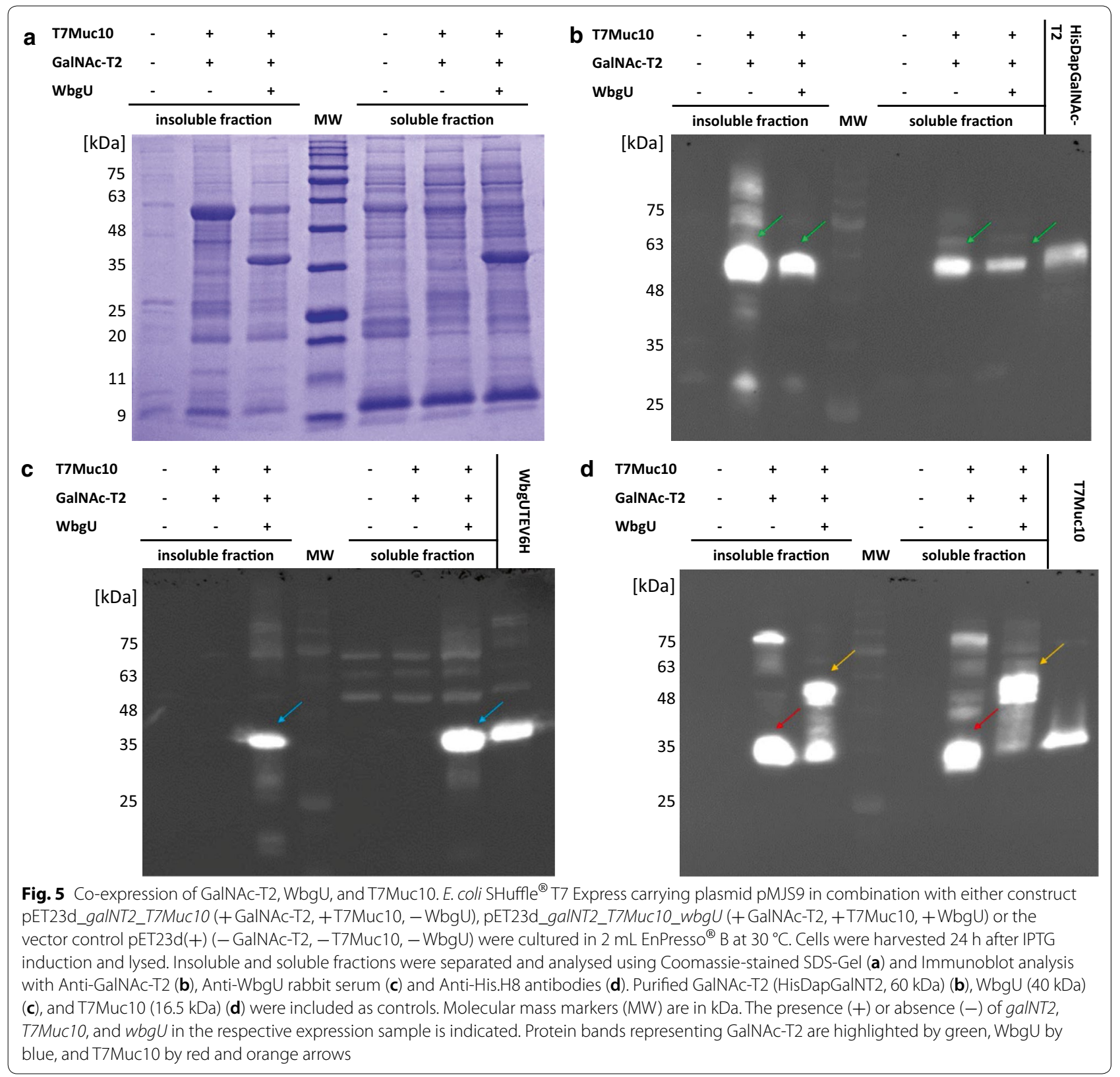

Evaluation of the MS/MS spectra revealed monosaccharide modifications at positions 2, 3, 6, 7, 11 and 12 of the tandem EA2. Monosaccharide modifications were also found at positions 2, 6, 7 and 11 of the EAN sequence. Interestingly, a single modified serine residue was also identified. This was detected at position 5 of the tandem EA2 sequence, adjacent to the asparagine- glycine linker (Additional file 1: Table S1).

\section{Discussion}

To establish an E. coli strain as cell factory for the production of recombinant glycoproteins, the functionality of the recombinant human GalNAc-T2 (shown in a previously described expression system) has been demonstrated in vitro [6]. In the present work, this expression strain was further modified to ensure the biosynthesis of the sugar substrate in the host background. The absence of the necessary activated sugar substrate (UDP-GalNAc) 


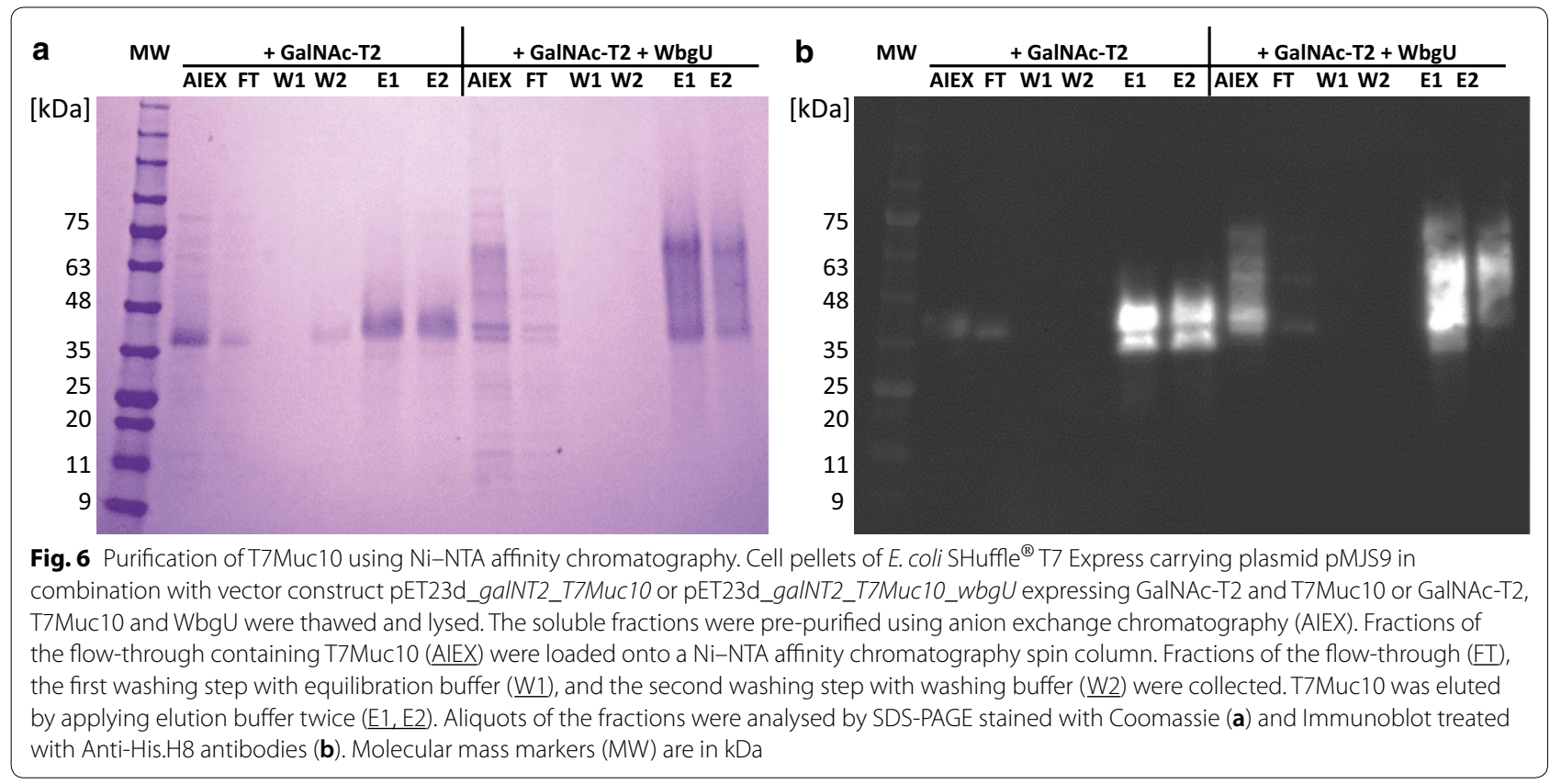

Table 1 Detected glycosylated and fragmented peptides of T7Muc10 using ESI-MS/MS

\begin{tabular}{lllll}
\hline Fragment sequence & $\begin{array}{l}\text { Peptide identification } \\
\text { probability [\%] }\end{array}$ & Observed $\mathbf{m} / \mathbf{z}$ & Actual peptide mass [Da] & $\begin{array}{l}\text { Position } \\
\text { of the modification } \\
\text { within the EA2 peptide }\end{array}$ \\
\hline PTTDSTTPAPTtK & 97 & 760.87 & 1519.73 & T12 \\
PTTDSTtPAPTTK & 97 & 760.87 & 1519.73 & $T 7$ \\
STTPAPTtKPT & 100 & 652.83 & 1303.65 & $T 12$ \\
STTPAPTTKPTtD & 100 & 760.87 & 1519.73 & $T 3$ \\
STTPAPtTKPTTD & 100 & 760.87 & 1519.72 & $T 11$ \\
\hline
\end{tabular}

T7Muc10 contains six repeating EA2 peptide sequences (PTTDSTTPAPTTK). To highlight adjacent EA2 repetitions within the detected fragments, amino acids of the neighbouring EA2 amino acid sequence are underlined. Identified modified threonines carrying GalNAc residues are displayed as lower-case letters in bold type

in laboratory $E$. coli strains requires the co-expression of an additional UDP-GlcNAc 4-epimerase to generate UDP-GalNAc from UDP-GlcNAc $[9,20]$. Therefore, we introduced the 4-epimerase $\mathrm{WbgU}$ into the previously described E. coli SHuffle ${ }^{\circledR} \mathrm{T} 7$ Express strain. The 4-epimerase was successfully expressed as judged by immunoblot analysis using polyhistidine-tag targeting antibodies. The protein was subsequently isolated and analysed. The in vitro activity of WbgU in combination with GalNAcT2 was demonstrated using a modified glycosyltransferase assay. As expected from previously published results [21], UDP-GlcNAc is not a substrate for GalNAcT2. Measured activities tended to be higher when WbgU and GalNAc-T2 were used in combination with UDPGalNAc. However, the comparison of the activities determined in this assay, whether in the presence of WbgU or not, is rather difficult to determine-due to the ongoing epimerization reaction. By adding the UDP-GlcNAc 4-epimerase to the assay, the ratio of UDP-GlcNAc:UDPGalNAc during the glycosylation reaction is unknown. In general, samples with both WbgU and UDP-GlcNAc showed comparable activities to samples with UDP-GalNAc, confirming the functionality of the 4-epimerase and the glycosyltransferases in the described assay in vitro.

The usefulness of E. coli SHuffle ${ }^{\circledR} \mathrm{T} 7$ Express for the expression of complex proteins containing disulfide bonds has already been demonstrated [6, 22, 23]. In vivo glycosylation has also been shown [9]. In the expression system published previously, an N-terminally truncated version of GalNAc T2, inserted under the control of the T7 promoter, has been expressed in combination with the 4-epimerase derived from $P$. aeruginosa including its endogenous promoter in E. coli SHuffle ${ }^{\circledR} \mathrm{T} 7$ Express without IPTG induction [9]. Glycosylation of a mucin fusion protein has been assessed by lectin in Western blot assays. The analysis of an anti-TNF- $\alpha$ antibody fragment 

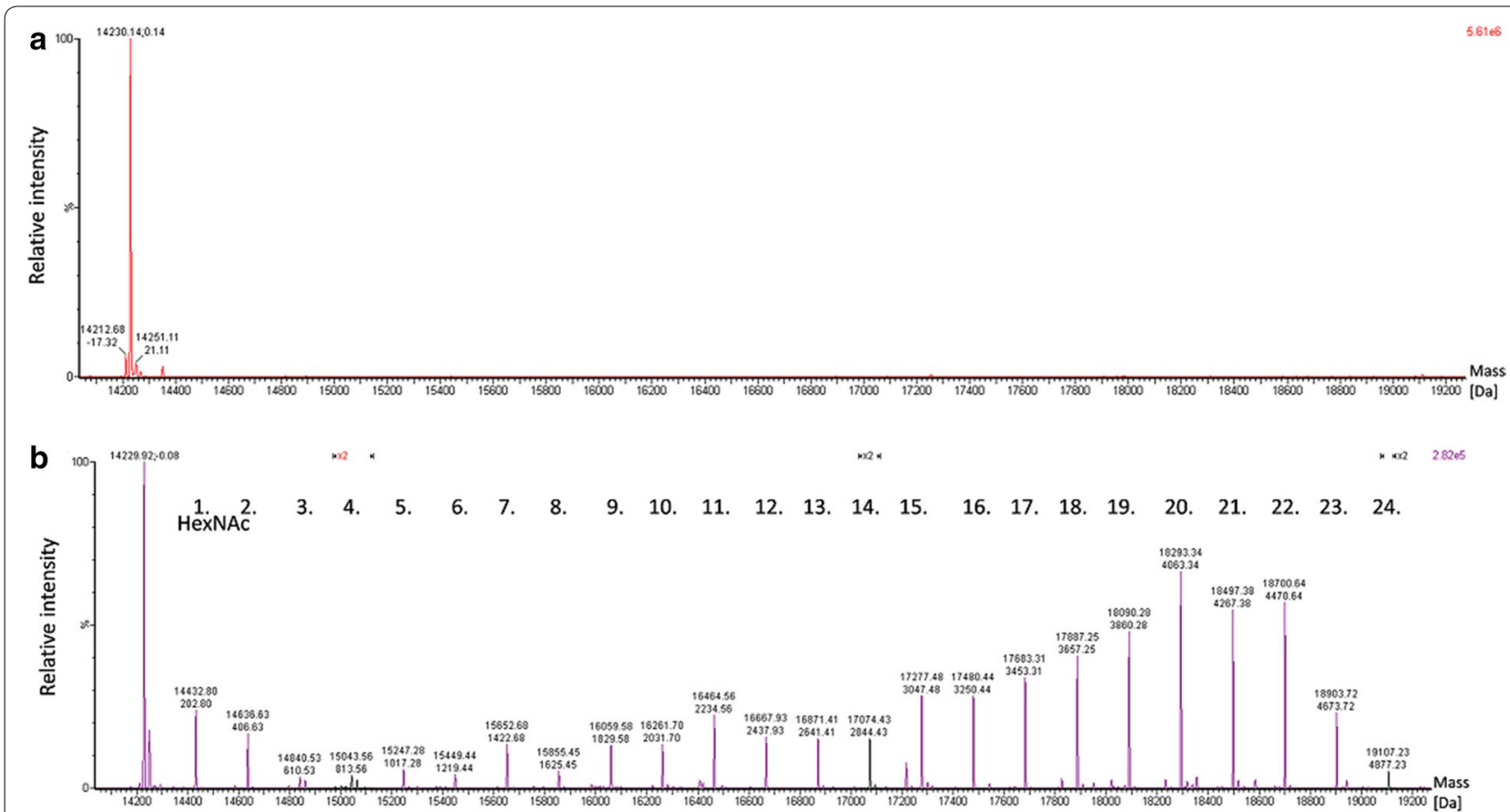

Fig. 7 UPLC-ESI-QTOF-MS analysis of intact unglycosylated and glycosylated T7Muc10. Purified protein samples were dialysed, filtered, and analysed by UPLC-ESI-QTOF mass spectrometry. Charge deconvolution of MS spectra was realized using the MaxEnt 1 algorithm. Charge-deconvoluted MS spectra of intact N-terminally truncated T7Muc10 (AS 21-159) (a) and glycosylated T7Muc10 (b) are shown. The average molecular mass ( $\mathrm{Da})$ of respective proteoforms is given. Each of the up to $24 \mathrm{O}$-linked $\beta$ - $\mathrm{N}$-acetylgalactosamines results in a mass increment of 203.2 Da

fusion to the 15 amino acid mucin-derived small peptide CDNKPAPGSTAPPAA by LC/MS revealed an increase in molecular weight of $203 \mathrm{Da}$, consistent with the presence of a single GalNAc moiety [9]. The present work describes a different approach to isolate a robust expression strain, aiming towards high-level in vivo glycosylation of complex proteins. The successful co-expression of GalNAc-T2 and WbgU- under the control of the T7 promotor in the presence of the two chaperones Erv1p/PDI in E. coli SHuffle ${ }^{\circledR} \mathrm{T} 7$ Express with IPTG and L-arabinose induction-was demonstrated by immunoblot analysis with Anti-GalNT2 antibodies and polyclonal Anti-WbgU serum. The potential of the $E$. coli strain was assessed by co-expressing the target protein T7Muc10, containing 60 putative glycosylation target sites, with 49 threonine- and 11 serine residues. In mammals, mucins form homooligomers via inter-molecular disulfide bonds between cysteine-rich domains at both ends of the proteins [24]. Using porcine submaxillary mucin (PSM) as an example, $\mathrm{N}$-glycosylation is followed by the dimerization of PSM via disulfide bond formation between the C-terminal domains [25-27]. Subsequently, O-glycosylation and $\mathrm{N}$-terminal multimerization of the dimers [25-27] occur, mediated by disulfide bonds between the $\mathrm{N}$-termini [27] Furthermore, non-covalent dimerization-probably by means of hydrophobic interactions-of domains of the colonic human MUC2 mucin containing cysteines has been identified [28]. For MUC5AC and MUC5B, higher molecular weight aggregates have been detected using SDS-PAGE, under both reducing and non-reducing conditions [29]. The synthetic T7Muc10 target protein used in the present work does not contain cysteine residues. As a result, the observed putative dimerization of the protein, indicated by the $33 \mathrm{kDa}$ protein band in SDSPAGE analysis, may be due to different interactions.

The potential glycosylation of T7Muc10 was subsequently verified by ESI-MS/MS. The results showed the presence of different glycosylation patterns with varying target sites and different amounts of monosaccharide moieties. These results are consistent with the presence of GalNAc residues attached to the glycosylated mucin. No molecular shift of T7Muc10 was detected when GalNAc-T2 and WbgU were not coexpressed. Putative glycosylation sites were postulated for di- or triglycosylated EA2 and EAN sequences based on MS1 measurements. These sites were confirmed by subsequent MS2 analysis. Although UDPgalactose has been postulated as an alternative sugar substrate for GalNAc-T2 [21, 30], no sugar moieties attached to T7Muc10, which correlate with galactose 
Table 2 HPLC-ESI-LTQ-OT-MS/MS analysis of glycosylated T7Muc10

\begin{tabular}{|c|c|c|c|c|c|c|c|c|}
\hline $\begin{array}{l}\text { Peptide } \\
\text { description }\end{array}$ & Peptide sequence & $\begin{array}{l}\text { Retention } \\
\text { time [min] }\end{array}$ & $\mathrm{m} / \mathrm{z}$ measured & {$[\mathrm{M}+\mathrm{H}]_{\text {theor }}^{+}$} & Charge state & $\Delta \mathrm{m}[\mathrm{ppm}]$ & $\begin{array}{l}\text { GalNAc } \\
\text { moieties }\end{array}$ & $\begin{array}{l}\text { Relative } \\
\text { abundance } \\
{[\%]}\end{array}$ \\
\hline \multirow[t]{2}{*}{1} & YTMAPTT & 16.93 & 784.355 & 784.355 & +1 & -0.98 & 0 & 88.21 \\
\hline & & 18.54 & 987.434 & 987.434 & +1 & 0.61 & 1 & 11.80 \\
\hline \multirow[t]{6}{*}{$2 / 3 / 4 / 7 / 8$} & DSTTPAPTTKPTT & 11.65 & 1317.653 & 1317.653 & +1 & 0.62 & 0 & 27.64 \\
\hline & & 10.68 & 1520.733 & 1520.733 & +1 & -0.40 & 1 & 40.35 \\
\hline & & 9.14 & 1723.812 & 1723.812 & +1 & 0.31 & 2 & 25.10 \\
\hline & & 10.83 & 1723.812 & 1723.812 & +1 & -0.32 & 2 & 4.34 \\
\hline & & 10.47 & 1926.891 & 1926.891 & +1 & -0.18 & 3 & 2.22 \\
\hline & & 10.03 & 1065.489 & 2129.971 & +2 & -0.37 & 4 & 0.36 \\
\hline \multirow[t]{7}{*}{$5 / 6$} & DSTTPAPTNKPTT & 10.42 & 1330.649 & 1330.649 & +1 & 0.23 & 0 & 21.00 \\
\hline & & 9.82 & 1533.728 & 1533.728 & +1 & 0.39 & 1 & 36.34 \\
\hline & & 8.38 & 1736.807 & 1736.807 & +1 & 0.13 & 2 & 27.71 \\
\hline & & 9.98 & 1736.807 & 1736.807 & +1 & -0.07 & 2 & 12.00 \\
\hline & & 8.00 & 1939.887 & 1939.887 & +1 & -0.01 & 3 & 2.45 \\
\hline & & 9.93 & 1939.887 & 1939.887 & +1 & -0.96 & 3 & 0.43 \\
\hline & & 9.47 & 1071.987 & 2142.966 & +2 & -0.14 & 4 & 0.07 \\
\hline 9 & $\begin{array}{l}\text { DSTTPAPTTKNNNNNNNNNG- } \\
\text { GLVPRGSGGGSGHHHHHH- } \\
\text { HHHH }\end{array}$ & n.d. & & 4339.9583 & & - & 0 & 0 \\
\hline
\end{tabular}

Proteolytic (AspN) peptides of glycosylated T7MuC10 were identified via HPLC-ESI-LTQ-OT-MS/MS analysis. The theoretical monoisotopic mass increases by 203.07937 u per attached O-acetylgalactosamine (C8O5NH13) moiety. Relative quantification of peptides was conducted using the MassMap ${ }^{\circledR}$ software. Extracted ion chromatograms (XIC) of MS signals were generated using a mass tolerance of 5 ppm. Relative peptide abundances of glycosylated and non-glycosylated peptides were calculated from the respective peptide peak areas. The C-terminal peptide was not detected (n.d.)

residues were detected. GalNAc-T2 has been classified as an early transferase, preferring mono- or unglycosylated protein substrates [14]. Previous in vitro studies with GalNAc-T2 have identified Thr7 in the synthetic EA2 peptide sequence $[14,15]$ and Thr9 in the MUC5AC peptide as preferred glycosylation sites [31]. Both threonines are located in the central areas of the peptide sequences. We have recently demonstrated the presence of up to three GalNAc residues attached by GalNAc-T2 to the EA2 peptide in vitro [6]. Differences in the respective results reflect the use of a short EA2 peptide in a chemically defined in vitro environment, as compared to the glycosylation of the larger artificial T7Muc10 derivative in a complex cytoplasmic environment in vivo. The data presented in this work indicates the truncated T7Muc10 glycosylation at different positions of the 57 possible target sites, in various degrees and combinations, leading to a multitude of different glycopeptides; thus demonstrating a high level mucin-type $O$-glycosylation. Furthermore, the results emphasize the potential of the presented glycosylation platform for in vivo glycosylation of commercially relevant biopharmaceuticals.

\section{Conclusion}

High level in vivo O-glycosylation of a MUC10 derived protein was demonstrated using the $E$. coli-based expression platform presented in this work. $O$-glycosylation sites were modified in different combinations, and to various degrees, by the co-expression of the target protein, the human glycosyltransferase GalNAc-T2, the 4-epimerase $\mathrm{WbgU}$, and the redox folding helper proteins PDI and Erv1p in E. coli SHuffle ${ }^{\circledR}$ T7 Express. The functionality of GalNAc-T2 and WbgU was also demonstrated in vitro, and the soluble expression of both enzymes was confirmed. Threonines at position 2, 3, 6, 7, 11 and 12 of the tandem EA2, correlating with threonines at position 2, 6, 7 and 11 of the EAN sequence, and a single serine residue at position 100 in the truncated T7Muc10, were identified as potential glycosylation sites in vivo. A maximum of 4 glycosylated sites in a peptide EA2/EAN repeat were detected. The value of the presented robust glycosylation platform will be further verified by introducing pharmaceutically relevant target proteins to assess in vivo glycosylation of important biopharmaceuticals. The approach might further enable the production of glycosylated protein based drugs that are easily accessible for site-specific in vitro polyethylene glycol modification (PEGylation), which would allow for the generation of long-lasting dosage forms in the future $[9,32]$. 


\section{Materials and methods \\ Bacterial strains}

The Escherichia coli strains NovaBlue (Novagen, Merck Millipore, Germany) and SHuffle ${ }^{\circledR} \mathrm{T} 7$ Express $(\mathrm{C} 3028 \mathrm{H}$, New England Biolabs, Germany) were routinely grown as described previously [6]. Transformation and selection were carried out following protocols already published [6]. Experiments to produce WbgU were carried out using TB Medium supplemented with $0.5 \%$ D-glucose (Glc). For the production of unglycosylated T7Muc10 5010 Medium (50 g/L yeast extract, $10 \mathrm{~g} / \mathrm{L}$ peptone, $0.492 \mathrm{~g} / \mathrm{L}$ magnesium sulfate heptahydrate) was used. Glycosylated T7Muc10 was expressed as described previously in a 24-deepwell plate containing $2 \mathrm{~mL}$ EnPresso ${ }^{\circledR} \mathrm{B}$ medium in each well and pooled for purification [6].

\section{Plasmids}

Plasmid DNA of pET-23a $(+)$ and pET-23d $(+)$ (both obtained from Merck Millipore (Germany)) was prepared using the Roti ${ }^{\circledR}$-Prep Plasmid MINI kit (Carl Roth GmbH \& Co. KG, Germany), DNA fragments were isolated from agarose gel blocks employing the Monarch Gel Extraction Kit (New England Biolabs, Germany) and PCR products were purified using the PCR Clean Up Extraction Kit (GeneOn, Germany). Primers were obtained from Thermo Scientific, Germany. Synthesised DNA fragments of $w b g U$ and muc10 were purchased from GeneArt ${ }^{\circledR}$ Gene Synthesis and supplied as shuffle vector pMA-T-derived constructs (Life Technologies GmbH, Germany). Plasmid pMJS9 [8] was kindly provided by Prof. Dr. L. W. Ruddock.

The DNA sequence encoding the UDP-GlcNAc 4-epimerase WbgU derived from Plesiomonas shigelloides [WbgU, GenBank:AAG17409.1, UniProtID:Q7BJX9] was adapted for expression in an Escherichia coli strain background [33]. The restriction sites $X b a \mathrm{I}$ and XhoI were added at the $5^{\prime}$ and $3^{\prime}$ end of the DNA fragment, respectively. Gene fragments were amplified by PCR using Primer 1 (CTC TCT AGA AAT AAT TTT), Primer 2 (ATT CTC GAG TGA ACC TTT CAG AAA ACG AAC), dNTP's (GeneOn, Germany) and Phusion Polymerase (Thermo Scientific, Germany). The stop codon TAA was replaced with the serine codon TCA to translationally fuse the His-tag sequence of the pET-23a vector. The isolated PCR product was digested with $\mathrm{XbaI}$ and $\mathrm{XhoI}$ and the resulting $1.1 \mathrm{~kb}$ fragment was purified and ligated with vector pET-23a treated with the same restriction enzymes. The DNA was subsequently used to transform E. coli NovaBlue and construct pET23a $(+) \_w b g U 6 x h i s$ was isolated encoding WbgU with the sequence SLE$\mathrm{HHHHHH}^{*}$ translationally fused to the C-terminal glycine. Annealed oligo cloning with the $5^{\prime}$-phosphorylated
Primer 3 (TCG AGA ACC TGT ATT TTC AGA GCG) and 4 (TCG ACG CTC TGA AAA TAC AGG TTC) was used to introduce the specific recognition sequence of the TEV protease into the XhoI restriction site of pET23a(+)_wbgU6xhis. Consequently, the C-terminus of WbgU was extended to SLENLYFQ'SVEHHHHHH*, with the apostrophe representing the cleavage site and the asterisk the stop codon. The resulting construct pET23a $(+) \_w b g U T E V 6 H$ was verified by restriction digests and DNA sequence analysis.

Plasmid pET23a_galNT2 was constructed by introducing galNT2 from the donor plasmid pMK-RQ [6] into pET23a(+) using NdeI and XhoI restriction sites. Ligation of the galNT2-fragment treated with $X b a \mathrm{I}$ and SpeI restriction endonucleases and the XbaI linearized $\mathrm{pET} 23 \mathrm{~d}(+)$ vector resulted in isolating construct pET23d_galNT2. The cloning steps to assemble vector pET23d_galNT2_T7Muc10_wbgU were carried out by Synbio Technologies LLC, NJ, USA, including the SacI/NotI and BamHI/EcoRI restriction digestion of the pMA-T vectors carrying $w b g U$ and muc10, respectively, and the ligation of the isolated DNA fragments encoding WbgU and Muc10 with the corresponding enzymesdigested vector fragment of pET23d_galNT2. The N-terminus of Muc10 was translationally fused to the T7-tag encoded on the pET23d_galNT2 vector resulting in T7Muc10. Plasmid pET23d_galNT2_T7Muc10 was obtained by deleting $w b g U$ from pET23d_galNT2_T7Mu c10_wbgU using EcoRI/XhoI restriction and 5'-phosphorylated Primer 5 (AAT TCC AAT TGT GAG CGG C) and 6 (TCG AGC CGC TCA CAA TTG G) to religate the vector.

The constructs were verified by restriction digests and the inserted fragments were confirmed by DNA sequence analysis.

\section{Expression of WbgU, HisDapGalNAc-T2, and T7Muc10}

For large-scale cultivation, a bioreactor BIOSTAT $^{\circledR}$ $\mathrm{C}$-DCU with a working volume of $10 \mathrm{~L}$ was used. Temperature, $\mathrm{pH}$, and aeration were set to $30{ }^{\circ} \mathrm{C}, \mathrm{pH} 7$, and $6 \mathrm{slpm}$, respectively. The oxygen saturation was kept constant at $60 \%$ via agitation starting with a lower limit of $100 \mathrm{rpm}$. The $\mathrm{pH}$ was adjusted with $1 \mathrm{M} \mathrm{NaOH}$ and $1 \mathrm{M} \mathrm{H} \mathrm{H}_{2} \mathrm{SO}_{4}$. E. coli SHuffle ${ }^{\circledR} \mathrm{T} 7$ Express harboring pET23a(+)_wbgUTEV6H and pMJS9 was pre-cultured in two baffled shake flasks with $750 \mathrm{~mL}$ TB medium at $30{ }^{\circ} \mathrm{C}$ and $140 \mathrm{rpm}$ until $\mathrm{OD}_{600}>2$ was reached. Subsequently, the bioreactor was inoculated and at $\mathrm{OD}_{600}$ of 1 a feed was started till a total volume of $20 \mathrm{~L}$. L-arabinose was added to a final concentration of $0.5 \%$. The culture was harvested $6.5 \mathrm{~h}$ post inoculation and the pellet with a wet cell weight of $257 \mathrm{~g}$ was stored at $-20^{\circ} \mathrm{C}$. 
Protein isolation of HisDapGalNAc-T2 was carried out using E. coli SHuffle ${ }^{\circledR} \mathrm{T} 7$ Express harboring pET23d(+)_HisDapGalNAcT2 [6] and pMJS9. The cells were pre-cultured in $5 \mathrm{~mL}$ LB medium supplemented with $0.5 \%$ Glc at $30{ }^{\circ} \mathrm{C}$ and $175 \mathrm{rpm}$. A baffled shaking flask with a working volume of $25 \%$ EnPresso ${ }^{\circledR}$ B medium was inoculated to $\mathrm{OD}_{600}$ of 0.01 and reagent $\mathrm{A}$ was added at a ratio of 1:2000. The culture was incubated at $30{ }^{\circ} \mathrm{C}$ and $170 \mathrm{rpm} .17 \mathrm{~h}$ post-inoculation booster tablets (1 per $50 \mathrm{~mL}$ ), L-arabinose (final concentration $0.5 \%$ ) and $17.5 \mathrm{~h}$ post-inoculation IPTG (final concentration $1 \mathrm{mM}$ ) were added. Cells from a $125 \mathrm{~mL}$ culture were harvested $24 \mathrm{~h}$ post-arabinose-induction and a pellet with a wet cell weight of $2.2 \mathrm{~g}$ was obtained and stored at $-20^{\circ} \mathrm{C}$.

To produce large amounts of unglycosylated T7Muc10, E. coli SHuffle ${ }^{\circledR} \mathrm{T} 7$ Express harboring pET23d(+)_galNT2_T7Muc10 and pMJS9 was pre-cultured in a $2 \mathrm{~L}$ baffled shaking flask with $1 \mathrm{~L} 5010$ medium at $30{ }^{\circ} \mathrm{C}$ and $160 \mathrm{rpm}$ until an $\mathrm{OD}_{600}$ higher than 2 was reached. Two $5 \mathrm{~L}$ baffled shaking flasks with $900 \mathrm{~mL} 5010$ medium were inoculated each with $350 \mathrm{~mL}$ cell culture and incubated at $30{ }^{\circ} \mathrm{C}$ and $170 \mathrm{rpm}$. When the $\mathrm{OD}_{600}$ was higher than 1, IPTG and L-arabinose were added with final concentrations of $1 \mathrm{mM}$ and $0.5 \%$, respectively. Cells were harvested $4 \mathrm{~h}$ post-induction and a pellet with a wet cell weight of $8.4 \mathrm{~g}$ was obtained and stored at $-20{ }^{\circ} \mathrm{C}$.

Glycosylated T7Muc10 was produced by cultivating $E$. coli SHuffle ${ }^{\circledR} \mathrm{T} 7$ Express harboring pET23d(+)_galNT2_ T7Muc10_wbgU and pMJS9 in two 24-deepwell plates filled with $2 \mathrm{~mL}$ EnPresso ${ }^{\circledR} \mathrm{B}$ medium per well and incubated at $30^{\circ} \mathrm{C}$ and $200 \mathrm{rpm}$. Expression experiments were carried out as described for HisDapGalNAc-T2. A pellet with a wet cell weight of $1.26 \mathrm{~g}$ was obtained and stored at $-20^{\circ} \mathrm{C}$.

\section{Protein purification}

$1 \mathrm{~g}$ of the respective expression culture cell pellet was resuspended in $2 \mathrm{~mL}$ extraction buffer $(50 \mathrm{mM}$ tris, $300 \mathrm{mM} \mathrm{NaCl}$; pH 8 for Ni-NTA affinity chromatography or $50 \mathrm{mM}$ tris $\mathrm{pH} 9$ for anion exchange chromatography) containing $112 \mu \mathrm{L}$ lysozyme $(10 \mathrm{mg} / \mathrm{mL})$ and $1 \mu \mathrm{L}$ DNAseI $(10 \mathrm{kU} / \mathrm{mL})$. The bacterial suspension was kept on ice for $2 \mathrm{~h}$ and sonicated for $2.5 \mathrm{~min}$ cooled on ice. The cell lysate was centrifuged at $4{ }^{\circ} \mathrm{C}, 18,000 \times g$ for $10 \mathrm{~min}$, the supernatant collected, $\mathrm{pH}$-value confirmed, and the liquid was subsequently passed through a $0.45 \mu \mathrm{M}$ filter (Titan $3^{\mathrm{TM}}$ PVDF, Life Technologies GmbH, Germany).

The polyhistidine-tagged proteins HisDapGalNAc-T2 and WbgUTEV6H were purified on a HisTrap HP $1 \mathrm{~mL}$ column with an ÄKTA Purifier system (GE Healthcare Life Sciences). The column was equilibrated with buffer
A1 (50 mM tris, $300 \mathrm{mM} \mathrm{NaCl}$; pH 8) and the sample was loaded. For HisDapGalNAc-T2 the column was washed with $22 \%$ buffer B1 (50 mM tris, $300 \mathrm{mM} \mathrm{NaCl}, 500 \mathrm{mM}$ imidazole; $\mathrm{pH} 8$ ) and the protein eluted with $55 \%$ buffer B1. Fractions containing HisDapGalNAc-T2 were dialysed (Slide-A-Lyzer ${ }^{\mathrm{TM}}$, Thermo Scientific, Rockford, IL) in $25 \mathrm{mM}$ tris, $150 \mathrm{mM} \mathrm{NaCl}$; $\mathrm{pH} 7.3$ and stored at $4{ }^{\circ} \mathrm{C}$. For WbgUTEV6H the column was washed with $10 \%$ and the protein eluted with $16 \%$ buffer B1. Fractions were collected during the elution procedure and samples containing WbgUTEV6H were pooled and dialysed against $50 \mathrm{mM}$ tris, $300 \mathrm{mM} \mathrm{NaCl}$; pH 8.0. To remove the polyhistidine tag, $2 \mu \mathrm{l}$ TEV protease (Protean s.r.o., Dobra Voda, Czech Republic) per $100 \mu$ g protein was added and subsequently incubated at $30{ }^{\circ} \mathrm{C}$ for $3 \mathrm{~h}$. Using Ni-NTA affinity chromatography, TEV protease-treated WbgU was obtained in the flow-through. Fractions containing WbgU were concentrated using Vivaspin ${ }^{\mathrm{TM}}$ columns (MWCO $10 \mathrm{kDa}, \mathrm{GE}$ Healthcare Life Sciences) and dialysed against $50 \mathrm{mM}$ Tris; $\mathrm{pH}$ 8.0. For long-term storage at $4{ }^{\circ} \mathrm{C}, 2 \times$ storage buffer $(50 \mathrm{mM}$ tris, $1 \mathrm{M}$ trimethylamine $N$-oxide, $1 \mathrm{M}$ trehalose; $\mathrm{pH} 8.0$ ) was added in a $1: 2$ ratio.

T7Muc10 was purified using anion exchange chromatography (AIEX). Four $1 \mathrm{~mL}$ CaptoAdhere columns (GE Healthcare Life Sciences) in serial arrangement were equilibrated with buffer A2 (50 mM tris; pH 9) and the sample was loaded. The protein was recovered in the fractions of the flow-through. The samples were pooled, titrated to $\mathrm{pH}$ 8.0, and concentrated using Ni-NTA affinity spin columns (Qiagen, Hilden, Germany), according to the manufacturer's instructions employing buffer A1 and B1. Concentrated fractions were pooled, dialysed against $20 \mathrm{mM}$ tris; $\mathrm{pH} 8.0$, and filtered $(0.2 \mu \mathrm{m}$, Phenomenex, Aschaffenburg, Germany).

\section{Analysis of purified protein}

SDS-PAGE and immunoblot analyses were carried out using standard protocols with dithiothreitol (DTT) as the reducing agent. Densitometric analysis was performed employing Fusion-FX software (Vilber Lourmat, Germany). For Western blot analysis, proteins were transferred to polyvinylidene fluoride membranes (PVDF, Bio-Rad Laboratories Inc., USA) and treated with mouse Anti-human GALNT2 antibodies (H00002590-A01, Acris Antibody GmbH, Germany), 6x-His tag monoclonal antibody HIS.H8 (Thermo Scientific, Rockford, IL) or Anti-WbgU serum (WbgU-immunized rabbits, Pineda antibody service, Berlin, Germany). Binding was detected with horseradish peroxidase (HRP)-labeled secondary antibodies (Jackson ImmunoResearch, West Grove, PA) 
and chemiluminescence was assessed using Clarity $^{\mathrm{TM}}$ or WesternBright ECL solutions.

Purified WbgU was analysed by circular dichroism (CD) spectroscopy to determine folding state and thermal transitions of the protein in the presence and absence of the activated sugar substrates UDP-GlcNAc and UDP-GalNAc (25 mM each). Spectra of the filtered solution (NanoSep ${ }^{\circledR}$ MF $0.2 \mathrm{mM}$, Pall) containing $0.6 \mathrm{mg} / \mathrm{mL}$ WbgU in $20 \mathrm{mM}$ sodium phosphate buffer (pH 7.75) were recorded in a $715 \mathrm{CD}$-spectropolarimeter (Jasco, Hachioji, Japan) at $25^{\circ} \mathrm{C}$. Spectra were measured from 190-260 nm with wavelength steps of $0.1 \mathrm{~nm}$ and a scan speed of $50 \mathrm{~nm}$ per minute. The averaged signal from four scans was corrected for the buffer signal. Thermal transitions were recorded at $220 \mathrm{~nm}$ with a step size of $0.1^{\circ} \mathrm{C}$ and a thermal slope of $1{ }^{\circ} \mathrm{C}$ per minute. The data was fitted using a Boltzmann equation $y=\frac{\mathrm{A}_{1}-\mathrm{A}_{2}}{1+e\left(x-x_{0}\right) / d x}+\mathrm{A}_{2}$ to obtain the transition point (OriginPro 8, OriginLab, Northampton, Massachusetts, USA).

For N-terminal Edman sequencing of $\mathrm{WbgU}$ and T7Muc10, solutions containing about $80 \mu \mathrm{g}$ of protein in $20 \mathrm{mM}$ sodium phosphate $\mathrm{pH} 7.75$ were supplied for sequencing (Proteome Factory, Berlin, Germany).

\section{Protein activity assays}

The substrate conversion of the sugar substrates UDPGlcNAc and UDP-GalNAc in the presence of WbgU was analysed by capillary electrophoresis (CE). $0.15 \mathrm{mM}$ and 1.5 mM UDP-HexNAc ( $N$-Acetylhexosamine) concentrations in $20 \mathrm{mM}$ tris; $\mathrm{pH} 8.5$ were incubated at $37^{\circ} \mathrm{C} .80 \mu \mathrm{l}$ samples were taken at 8 different time points $(0,1,2,3$, $5,7,10$ and $15 \mathrm{~min}$ ) and inactivated at $65{ }^{\circ} \mathrm{C}$ for $5 \mathrm{~min}$. After the first sampling, $3.5 \mu \mathrm{g} \mathrm{WbgU}$ was added to the remaining $560 \mu \mathrm{L}$ reaction volume and subsequently, samples were taken at the next 7 time points. Heat inactivated samples were stored at $4{ }^{\circ} \mathrm{C}$. Prior to analysis, samples were centrifuged. UDP-GlcNAc and UDP-GalNAc were separated employing the CE-UV 7100 system (Agilent Technologies, Santa Clara, CA), equipped with a fused silica capillary $(50 \mu \mathrm{m}$ inside diameter, $65 \mathrm{~cm}$ total length and $56.5 \mathrm{~cm}$ effective separation length), and using a $20 \mathrm{mM}$ sodium borate, $64 \mathrm{mM}$ boric acid; $\mathrm{pH} 9.0$ buffer system at $30 \mathrm{kV}$. Samples were injected at $30 \mathrm{mbar}$ for $10 \mathrm{~s}$ and detected at $254 \mathrm{~nm}$. Monitored data was evaluated with openLAB CDS CehmStation C.01.07.

The activity of WbgU in combination with HisDapGalNAc-T2 and rhGalNAc-T2 (7507-GT-020, R\&D Systems Europe Ltd., UK) was monitored using a modified version of the previously described glycosyltransferase activity assay (EA001, R\&D Systems Europe Ltd., UK) [6] by replacing UDP-GalNAc with UDP-GlcNAc and $\mathrm{WbgU}(1 \mu \mathrm{g})$. Solutions containing $\mathrm{WbgU}$ were incubated for $20 \mathrm{~min}$ at $37{ }^{\circ} \mathrm{C}$ prior to the addition of glycosyltransferases.

\section{Mass spectrometry analysis}

After separation by SDS-PAGE and visualization of the proteins by Coomassie staining, protein bands containing T7Muc10 and potentially glycosylated T7Muc10 were cut out and submitted to the life science center of the University of Hohenheim (Germany) for protein identification via LC-ESI-MS/MS analysis. Excised gel pieces were in-gel digested with trypsin prior to LC-ESI-MS/ MS analysis.

Alternatively, purified T7Muc10 and potentially glycosylated T7Muc10 were subjected to in-solution digestion. Sequencing grade Asp-N (Promega Corp., Madison, WI) was added to a final enzyme:substrate ratio of 1:100 (w/w) and the samples were incubated for $18 \mathrm{~h}$ at $37^{\circ} \mathrm{C}$. The resulting peptide mixtures were analysed by LC-MS on a Shimadzu Prominence HPLC system (Shimadzu, Kyoto, Japan) coupled to an LTQOrbitrap XL mass spectrometer (Thermo Fisher Scientific) equipped with a heated electrospray ionization source (Thermo Fisher Scientific). Chromatographic separation was carried out on a Symmetry C18 column $(2.1 \mathrm{~mm} \times 150 \mathrm{~mm}, 3.5 \mu \mathrm{m}, 100 \AA$ A, Waters Corp., Milford, MA). Peptide elution was performed by applying a mixture of solvents $A$ and B. Solvent A consisted of $0.1 \%(\mathrm{v} / \mathrm{v})$ formic acid and solvent B was $0.1 \%(\mathrm{v} / \mathrm{v})$ formic acid in $85 \%(\mathrm{v} / \mathrm{v})$ acetonitrile (MS grade, Honeywell). Separations were performed by applying a linear gradient of $2 \%$ to $30 \%$ solvent B over $45 \mathrm{~min}$. The column was kept at $40{ }^{\circ} \mathrm{C}$ and the flow rate was set to $250 \mu \mathrm{l} / \mathrm{min}$. MS data were acquired throughout the duration of the gradient using the data-dependent MS/ MS mode. Each high-resolution full scan $(\mathrm{m} / z$ of 200 2000 and resolution of 60,000 ) in the Orbitrap analyzer was followed by five product ion scans (collisioninduced dissociation-MS/MS) in the linear ion trap for the five most intense signals of the full scan mass spectrum (isolation window, $2.0 \mathrm{Th}$ ). Dynamic exclusion (repeat count: 1, repeat duration: $30 \mathrm{~s}$; exclusion duration: $30 \mathrm{~s}$ ) was enabled to allow MS/MS-analysis of less abundant precursor ions. Peptide identification was performed using the Proteome Discoverer 1.4 (Thermo Fisher Scientific). MS and MS/MS data of precursor ions in the $m / z$ range 500-5000 were searched against an in-house database using SEQUEST. Mass accuracy was set to $5 \mathrm{ppm}$ and $0.8 \mathrm{Da}$ for precursor and fragment ions, respectively. Label-free relative quantification of peptides was conducted using the MassMap ${ }^{\circledR}$ software (MassMap GmbH \& Co. KG, Wolfratshausen, Germany). For all peptides of interest, extracted ion chromatograms (XIC) of MS signals were generated using a 
mass tolerance of $5 \mathrm{ppm}$. Relative peptide abundances of glycosylated and non-glycosylated peptides were calculated from the respective peptide peak areas.

Intact mass determination of T7Muc10 and potentially glycosylated T7Muc10 in $20 \mathrm{mM}$ tris $\mathrm{pH} 8.0$ was performed on an ACQUITY UPLC System (Waters Corp., Milford, MA) coupled to a Xevo G2 QTof mass spectrometer (Waters Corp., Milford, MA) equipped with an ESI source (ZSpray, Waters Corp., Milford, MA). Reversephase desalting and protein separation was carried out on a BioSuite ${ }^{\mathrm{TM}}$ Phenyl column (1000 $\AA, 10 \mu \mathrm{m}, 2.0 \mathrm{~mm}$ $\times 75 \mathrm{~mm}$, Waters Corp., Milford, MA). The mobile phase A was $0.1 \%(\mathrm{v} / \mathrm{v})$ formic acid in water, while mobile phase B contained $0.1 \%(\mathrm{v} / \mathrm{v})$ formic acid in acetonitrile. The flow rate and column temperature were maintained at $0.150 \mathrm{~mL} / \mathrm{min}$ and $65{ }^{\circ} \mathrm{C}$, respectively, throughout the run. Chromatographic separation was performed by applying a linear gradient from 10 to $35 \%$ solvent B over 15 min. Mass spectrometric analysis was carried out in positive ion mode. The desolvation gas and source temperatures were set to $240{ }^{\circ} \mathrm{C}$ and $115^{\circ} \mathrm{C}$, respectively. The capillary and cone voltages were set at 3000 and $35 \mathrm{~V}$, respectively. The instrument was calibrated in the $\mathrm{m} / \mathrm{z}$ range of 400-4000 using NaI. The deconvolution of ESI mass spectra of intact protein was performed by MassLynx V4.1 (Waters Corp., Milford, MA) using the MaxEnt 1 algorithm. For deconvolution, the input mass range from $m / z 500-2000$ was used with the following MaxEnt 1 parameters: output mass range from 5000 to $55,000 \mathrm{Da}$; minimum intensity ratio left and right, $50 \%$; width at half height for uniform Gaussian model, 0.6; number of iterations, 20.

\section{Additional file}

Additional file 1. HPLC-ESI-LTQ-OT-MS/MS analysis of glycosylated T7Muc 10. Proteolytic (AspN) peptides of glycosylated T7MuC10 detected using HPLC-ESI-LTQ-OT-MS analysis were further fragmented. To highlight adjacent EA2 (PTTDSTTTPAPTTK) or EAN (PTTDSTTTPAPTNK) repetitions within the detected fragments, amino acids of the neighbouring EA2 or EAN amino acid sequence are underlined. Identified modified threonines and serines carrying GalNAc residues using Proteome Discoverer 1.4 are lowercased and marked bold. The posterior error probability (PEP, the lower the better) and cross correlation (Xcorr, the higher the better) were included to evaluate the quality of the generated results.

\section{Authors' contributions}

PM contributed to the design of the study, performed experimental work, and participated in data analysis. SG and RH contributed to the conception and design of the study. The project was in close collaboration with RG and BE. NR was involved in the design of the artificial MUC10 derivative. SL performed the CD spectroscopy measurements. CW performed the capillary electrophoresis measurements, supported by LE. MK and MS performed mass spectrometry analysis supported by MZ. All authors made contributions to the writing of the manuscript. All authors read and approved the final manuscript.

\section{Author details}

${ }^{1}$ Institute of Applied Biotechnology, Biberach University of Applied Sciences, Biberach, Germany. ${ }^{2}$ Institute of Microbiology and Biotechnology, University of Ulm, Ulm, Germany. ${ }^{3}$ Institute for Biotechnology and Helmholtz-Institute for Biomedical Engineering, RWTH Aachen University, Aachen, Germany. ${ }^{4}$ Zhejiang University-Edinburgh University Institute, School of Medicine, Zhejiang University, Zhejiang, China. ${ }^{5}$ Boehringer Ingelheim Pharma GmbH and Co.KG, Analytical Development Biologics, Biberach, Germany.

\section{Acknowledgements}

We thank the International Graduate School in Molecular Medicine of UIm University (Germany) for scientific encouragement and financial support. We also thank the students Tanja Gehrig, Sonja Hartinger, Agnieszka Luzar, Philipp Brosig and Philipp Haas for their technical assistance. Special thanks goes to Don Crawford, Georgia Fodelianaki, Dr. Gillian Fraser, and Dr. Goeran Huebner for their support and valuable comments on the manuscript. Furthermore, we would like to express our gratitude to Prof. Dr. Lloyd W. Ruddock for providing the project with essential plasmids.

\section{Competing interests}

The authors declare that they have no competing interests.

\section{Availability of data and materials}

All data generated during this study is included in this article and the supplementary data.

\section{Consent for publication}

All authors read and approved the final manuscript.

\section{Ethics approval and consent to participate}

Not applicable.

\section{Funding}

This study was funded by the Cooperative Research Training Group Pharmaceutical Biotechnology as stated by the Postgraduate Scholarships Act of the Ministry for Science, Research and Arts of the federal state government of Baden-Württemberg, Germany.

\section{Publisher's Note}

Springer Nature remains neutral with regard to jurisdictional claims in published maps and institutional affiliations.

Received: 16 August 2018 Accepted: 19 October 2018

Published online: 26 October 2018

\section{References}

1. Walsh G. Therapeutic insulins and their large-scale manufacture. Appl Microbiol Biotechnol. 2005;67:151-9.

2. Wells EA, Robinson AS. Cellular engineering for therapeutic protein production: product quality, host modification, and process improvement. Biotechnol J. 2017;12:1600105.

3. Walsh G, Jefferis R. Post-translational modifications in the context of therapeutic proteins. Nat Biotechnol. 2006;24:1241-52.

4. Walsh G. Biopharmaceutical benchmarks 2014. Nat Biotech. 2014;32:992-1000

5. Lobstein J, Emrich CA, Jeans C, Faulkner M, Riggs P, Berkmen M. SHuffle, a novel Escherichia coli protein expression strain capable of correctly folding disulfide bonded proteins in its cytoplasm. Microb Cell Fact. 2012;11:56.

6. Lauber J, Handrick R, Leptihn S, Durre P, Gaisser S. Expression of the functional recombinant human glycosyltransferase GalNAcT2 in Escherichia coli. Microb Cell Fact. 2015;14:3.

7. Hatahet F, Ruddock LW. Protein disulfide isomerase: a critical evaluation of its function in disulfide bond formation. Antioxid Redox Signal. 2009;11:2807-50.

8. Nguyen VD, Hatahet F, Salo KE, Enlund E, Zhang C, Ruddock LW. Preexpression of a sulfhydryl oxidase significantly increases the yields of 
eukaryotic disulfide bond containing proteins expressed in the cytoplasm of E. coli. Microb Cell Fact. 2011;10:1.

9. Henderson GE, Isett KD, Gerngross TU. Site-specific modification of recombinant proteins: a novel platform for modifying glycoproteins expressed in E. coli. Bioconjug Chem. 2011;22:903-12.

10. Wacker M, Linton D, Hitchen PG, Nita-Lazar M, Haslam SM, North SJ, Panico M, Morris HR, Dell A, Wren BW, Aebi M. N-linked glycosylation in Campylobacter jejuni and its functional transfer into E. coli. Science. 2002:298:1790-3.

11. Valderrama-Rincon JD, Fisher AC, Merritt JH, Fan YY, Reading CA, Chhiba K, Heiss C, Azadi P, Aebi M, DeLisa MP. An engineered eukaryotic protein glycosylation pathway in Escherichia coli. Nat Chem Biol. 2012;8:434-6.

12. Fisher AC, Haitjema CH, Guarino C, Celik E, Endicott CE, Reading CA, Merritt JH, Ptak AC, Zhang S, DeLisa MP. Production of secretory and extracellular N-linked glycoproteins in Escherichia coli. Appl Environ Microbiol. 2011;77:871-81.

13. Kowarik M, Young NM, Numao S, Schulz BL, Hug I, Callewaert N, Mills DC, Watson DC, Hernandez M, Kelly JF, et al. Definition of the bacterial N-glycosylation site consensus sequence. EMBO J. 2006;25:1957-66.

14. Pratt MR, Hang HC, Ten Hagen KG, Rarick J, Gerken TA, Tabak LA Bertozzi CR. Deconvoluting the functions of polypeptide $\mathrm{N}-\mathrm{a}$ acetylgalactosaminyltransferase family members by glycopeptide substrate profiling. Chem Biol. 2004;11:1009-16.

15. Fritz TA, Raman J, Tabak LA. Dynamic association between the catalytic and lectin domains of human UDP-GalNAc:polypeptide alpha-N-acetylgalactosaminyltransferase-2. J Biol Chem. 2006;281:8613-9.

16. Chugh S, Gnanapragassam VS, Jain M, Rachagani S, Ponnusamy MP, Batra SK. Pathobiological implications of mucin glycans in cancer: sweet poison and novel targets. Biochim Biophys Acta. 2015;1856:211-25.

17. Kowal P, Wang PG. New UDP-GICNAC C4 epimerase involved in the biosynthesis of 2-acetamino-2-deoxy-l-altruronic acid in the $\mathrm{O}$-antigen repeating units of Plesiomonas shigelloides O17t. Biochemistry. 2002:41:15410-4.

18. Pey AL, Padín-Gonzalez E, Mesa-Torres N, Timson DJ. The metastability of human UDP-galactose $4^{\prime}$-epimerase (GALE) is increased by variants associated with type III galactosemia but decreased by substrate and cofactor binding. Arch Biochem Biophys. 2014;562:103-14.

19. Wu ZL. Phosphatase-coupled universal kinase assay and kinetics for firstorder-rate coupling reaction. PLOS ONE. 2011;6:e23172.

20. Merritt JH, Ollis AA, Fisher AC, DeLisa MP. Glycans-by-design: engineering bacteria for the biosynthesis of complex glycans and glycoconjugates. Biotechnol Bioeng. 2013:110:1550-64.

21. Wandall HH, Hassan $H$, Mirgorodskaya E, Kristensen AK, Roepstorff $P$, Bennett EP, Nielsen PA, Hollingsworth MA, Burchell J, Taylor-Papadimitriou J, Clausen H. Substrate specificities of three members of the human
UDP-N-acetyl-alpha-D-galactosamine: polypeptide $\mathrm{N}$-acetylgalactosaminyltransferase family, GalNAC-T1, -T2, and -T3. J Biol Chem. 1997:272:23503-14.

22. Robinson MP, Ke N, Lobstein J, Peterson C, Szkodny A, Mansell TJ, Tuckey C, Riggs PD, Colussi PA, Noren CJ, et al. Efficient expression of full-length antibodies in the cytoplasm of engineered bacteria. Nat Commun. 2015;6:8072.

23. Yao M, Wang J, Wu L, Min L, Li W-Y, Zhu L-Y, Meng E, Zhang D-Y. An efficient strategy for the expression of Jingzhaotoxin-III in Escherichia coli. Biotechnol Biotechnol Equip. 2017;31:821-7.

24. McGuckin MA, Linden SK, Sutton P, Florin TH. Mucin dynamics and enteric pathogens. Nat Rev Microbiol. 2011;9:265-78.

25. Perez-Vilar J, Hill RL. The carboxyl-terminal 90 residues of porcine submaxillary mucin are sufficient for forming disulfide-bonded dimers. J Biol Chem. 1998;273:6982-8.

26. Perez-Vilar J, Eckhardt AE, DeLuca A, Hill RL. Porcine submaxillary mucin forms disulfide-linked multimers through its amino-terminal D-domains. J Biol Chem. 1998;273:14442-9.

27. Perez-Vilar J, Hill RL. Identification of the half-cystine residues in porcine submaxillary mucin critical for multimerization through the D-domains. Roles of the CGLCG motif in the D1- and D3-domains. J Biol Chem. 1998;273:34527-34.

28. Ambort $\mathrm{D}$, van der Post $\mathrm{S}$, Johansson ME, Mackenzie J, Thomsson $\mathrm{E}$, Krengel U, Hansson GC. Function of the CysD domain of the gel-forming MUC2 mucin. Biochem J. 2011;436:61-70.

29. Backstrom M, Ambort D, Thomsson E, Johansson ME, Hansson GC. Increased understanding of the biochemistry and biosynthesis of MUC2 and other gel-forming mucins through the recombinant expression of their protein domains. Mol Biotechnol. 2013;54:250-6.

30. Gomez H, Rojas R, Patel D, Tabak LA, Lluch JM, Masgrau L. A computational and experimental study of O-glycosylation. Catalysis by human UDP-GalNAc polypeptide: GalNAc transferase-T2. Org Biomol Chem. 2014;12:2645-55.

31. Tetaert D, Ten Hagen KG, Richet C, Boersma A, Gagnon J, Degand P. Glycopeptide $\mathrm{N}$-acetylgalactosaminyltransferase specificities for O-glycosylated sites on MUC5AC mucin motif peptides. Biochem J. 2001;357:313-20.

32. Hoggatt J, Tate TA, Pelus LM. Role of lipegilgrastim in the management of chemotherapy-induced neutropenia. Int J Nanomedicine. 2015;10:2647-52

33. Krishna Rao DV, Rao JV, Narasu ML, Bhujanga Rao AKS. Optimization of the AT-content of codons immediately downstream of the initiation codon and evaluation of culture conditions for high-level expression of recombinant human G-CSF in Escherichia coli. Mol Biotechnol. 2008:38:221-32.
Ready to submit your research? Choose BMC and benefit from:

- fast, convenient online submission

- thorough peer review by experienced researchers in your field

- rapid publication on acceptance

- support for research data, including large and complex data types

- gold Open Access which fosters wider collaboration and increased citations

- maximum visibility for your research: over $100 \mathrm{M}$ website views per year

At $\mathrm{BMC}$, research is always in progress.

Learn more biomedcentral.com/submissions 